\title{
Audible Information Design in the New York City Subway System: A Case Study
}

\author{
Benjamin U. Rubin \\ Interactive Telecommunications Program, New York University \\ New York, USA
}

\begin{abstract}
Beginning with a detailed presentation of the use of audible signals, in the New York City subway stations and trains, we present an analysis of the information that is communicated by the existing sound design. We show the results of a survey of subway riders regarding their awareness and comprehension of the audible signals in the system. An analysis of the system's sound and information environment is presented, followed by a proposed sonic re-design to better serve both communication and aesthetic needs. We conclude by proposing that the principles of information design, sound design and music must be considered equally with those of acoustics and psychoacoustics when designing audio information and feedback systems.
\end{abstract}

\section{Introduction}

This paper presents a case study of the New York City subway system as a means to articulate a new methodology for the analysis, simulation, and design of sound information systems. It is a hypothetical exercise, in that the new design we propose for the subway system is not intended for immediate application and would not be practical to implement using the existing equipment.

While the New York City subway system is not a complex information environment, it nonetheless provides a very attractive site in which to study sound used as an information medium. At present, the use of sound is uniform across the hundreds of stations and thousands of trains throughout the system. More importantly, an audible signal is a crucial modality for the communication of several key pieces of information, including the success or failure of a turnstile transaction, and the arrival of a train in the station.

The New York City subway system thus presents some straightforward audible display design challenges: a few simple pieces of information need to be communicated quickly, unambiguously, and harmoniously by a small number of audible signals. How successful is the existing design at accomplishing this task, and how can it be improved? As we will see below, the existing design fails to reach its potential for informing system users through sound. The design also has important ergonomic and aesthetic shortcomings that will be discussed in greater detail below.

Principles from music, information design, interface design, and the emerging field of auditory display science are applied to create a new system-wide sound design. This proposed design will be presented using audiovisual simulations. Our goal is to explore the notion that a unified, musically composed sound design not only improves the experience of subway system users, but also expands the possibilities for communicating critical information through sound.

\section{Existing Audio Signals in the New York City Subway System}

All subway stations posses at least one row of turnstiles, a token booth where subway tokens and Metrocards (electronic fare cards) are sold, and a train platform (track) area. Any station in which the token booth and turnstiles are not immediately adjacent to the track also has a designated "Off-hours waiting area" that is generally within sight of the token booth and turnstiles.

\subsection{Turnstiles}

The New York City subway system uses both token coins and newer electronic farecards (called "Metrocards") at its turnstiles. Table 1 shows the various conditions and signals associated with turnstile transactions. The turnstiles display short electronic text messages that visually indicate the specific user condition, and there is a small speaker inside the card reader that beeps audibly after each card swipe. Example 1: turnstile signals is a 
recording of the single, double, and triple beep sounds produced by the turnstiles. ${ }^{1}$

\begin{tabular}{|l|l|l|}
\hline Transaction result & Specific user condition & Associated Sound \\
\hline \multirow{4}{*}{ Success } & Token OK & \multirow{2}{*}{ Plain beep ${ }^{2}$} \\
\cline { 2 - 2 } & Metrocard OK & \\
\cline { 2 - 2 } & Metrocard OK - reduced rate & Triple beep \\
\cline { 2 - 2 } & $\begin{array}{l}\text { Metrocard OK - funds low (one ride or } \\
\text { less remaining on pay-per-use Metrocard) }\end{array}$ & \\
\hline Swipe failure & Swipe again & Double beep \\
\cline { 2 - 2 } & Swipe again at this turnstile & \\
\hline Card failure & Insufficient funds on Metrocard & \\
\cline { 2 - 2 } & $\begin{array}{l}\text { Monthly, weekly, or daily Metrocard } \\
\text { expired }\end{array}$ & \\
\hline
\end{tabular}

Table 1: Existing mapping of turnstile conditions to audible signals

\subsection{Waiting Area}

All major subway stations and many smaller ones have waiting areas that are separate from the track platforms. Electronic display signs have been installed in these waiting areas to tell riders when a train is arriving. A text message on the sign indicates the destination of the train and its track designation (local or express). The text display is accompanied by a loud, pulsing whistle sound ${ }^{3}$ that is the same for all arriving trains. The sound can be heard in Example 2: arriving train signal.

While these electronic display signs are visible only in the area immediately in front of them, their signals are audible for hundreds of yards through stairways, connecting corridors, and on the train platforms. Many riders respond to the beep signal by breaking into a run in corridors or stairwells in hopes of catching the arriving train, even though the beep does not tell them the direction of the arriving train. Rider responses will be explored in more detail in section three below.

\subsection{Train Platforms and Interiors}

On train platforms, verbal announcements convey information about train delays or routing anomalies when necessary. No attention signal precedes these messages, nor are any other non-verbal audio signals routinely present here. Verbal announcements are also made aboard trains: as a train pulls into each station, the conductor announces the name of the station and any available connections. As the train prepares to leave a station, the conductor announces the next stop and recites a verbal warning to, "Stand clear of the closing doors," at which time a two-tone alert signal is sounded just prior to the doors closing. Example 3: subway train interior presents the audible signal heard inside the train cars.

\section{User Survey}

This section presents the responses of subway riders to the existing sound environment. Thirty-six subway riders were asked questions about their awareness of and feelings about the signals present in the system. While this survey is too small to be of statistical value, the rider responses nonetheless help to identify some shortcomings of the system and offer clues as to directions to follow in the redesign. Riders were asked questions about the

\footnotetext{
${ }^{1}$ Audio examples are available via the worldwide web at http://www.earstudio.com/research/subway.html or by contacting the author via e-mail at benrubin@earstudio.com.

${ }^{2}$ The "beep" sound is a short, constant tone with a fundamental frequency of about $2900 \mathrm{~Hz}$ and a 3rd harmonic at about $8700 \mathrm{~Hz}$ (the frequency varies from turnstile to turnstile by as much as $+/-50 \mathrm{~Hz}$ ). A single beep has a duration of one second, while the double beep consists of two $500 \mathrm{~ms}$ beeps separated by a $50 \mathrm{~ms}$ pause, and the triple beep consists of three 333 ms beeps separated by two 50 ms pauses.

${ }^{3}$ This warbling whistle or chip sound has frequency strong components at $2850 \mathrm{~Hz}, 2910 \mathrm{~Hz}$ and $3251 \mathrm{~Hz}$. The sound is pulsed in $1500 \mathrm{~ms}$ cycles $(750 \mathrm{~ms}$ on, $750 \mathrm{~ms}$ off). The entire signal consists of 8 pulses, and has a total duration of 12 seconds.
} 
helpfulness of three main signals in the system: the beeps of the turnstiles, the "train arriving" signal, and the "doors closing" signal. Figure 1 shows a summary of the results.

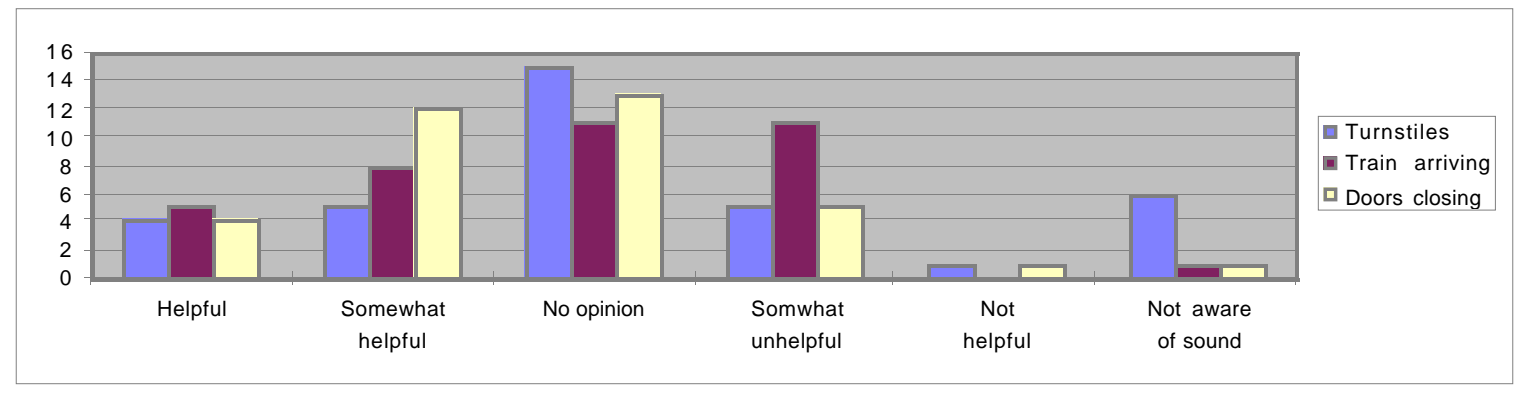

Figure 1: Summary of rider survey results

Respondents were also encouraged to offer opinions and observations about the quality of the sonic experience in the subway system. Regarding the turnstile beeps, four people said they could not tell the difference between the signals for successful and unsuccessful transactions. One rider said, "I hear a sound but I don't know what it means, so I just go through the turnstile and see if it works." Another person specifically commented that he liked the beeps and noticed that they meant different things. He especially appreciated the warning that his card was low on funds. Several people seemed to equate the "swipe again" signals with the fact that the Metrocards don't work well much of the time. Four of these people used the word "annoying" to describe this signal.

In response to the "train arriving" signal, seven people commented that you cannot tell which train is coming from the sound. Five people said that the signal is hard to distinguish from the combined sound of many Metrocards being swiped at the turnstiles. One woman commented, "It took me five years to figure out what the hell that sound is." Another person said the arrival beeps are too loud.

As for the "closing door" signal, five people commented that this sound made them feel safer. Six people called the sound annoying. Several people associated this sound with rush hour and stressful commutes. One person said, "I like it. It keeps me awake in the morning." Another person liked the musicality of it. One person was particularly enthusiastic about all the system's audible signals. "It's like Pavlonian conditioning," he said. "[The sounds] tell me my morning is going well."

\section{$4 \quad$ Analysis of the Existing Sound Design}

\subsection{Effective Communication}

The subway system's sound implementation fails to meet its potential for effective communication in several ways. Overall, there is a lack of coordination among the system's various sound components. This lack of coordination is evidenced, for example, by the masking effect of the train arrival signal by the turnstile beeps, a predictable result of using sounds within the same 1/3 octave frequency range [4].

As many of the riders surveyed noted, the turnstile beep sounds are indistinct, and the three different signals (single, double, and triple beep) are difficult to distinguish from each other. This may be due in part to the acoustic qualities of the signal, which is a relatively long, unmodulated, steady-state tone with virtually no onset transients in its attack. In the highly reverberant environment of the subway stations, these sounds are difficult to locate directionally, and at busy times, when many turnstiles are sounding at once, the individual beeps seem to merge into a continuos ringing tone. Once this state is reached, it is nearly impossible for a rider to distinguish the sound of his or her own turnstile from the ones around it, and the communication effectiveness of the signal is almost entirely lost.

In addition, the turnstile sounds are poorly mapped to the set of conditions they address. As we saw in table 1, above, there are three main possible outcomes of a Metrocard swipe, each requiring a different action on the part of the rider: success (the rider advances though the turnstile), swipe failure (the rider must swipe again), and card failure (the rider must step back out of the turnstile and obtain a new Metrocard). There are three sounds in the existing turnstile implementation, but two of them are mapped to success conditions, while the third is mapped to both swipe failure and card failure.

The "train arriving" signal is undifferentiated in its signalling the arrival of different trains at the station, 
missing an opportunity to inform commuters of whether to rush or relax as they approach the turnstiles. In part 5 , we will explore ways of differentiating this signal and of intuitively mapping the variants to the different train arrivals.

\subsection{Quality of Experience}

Despite its use of tonal signals, the New York City subway system sounds decidedly unmusical. The three different signals (turnstile, train arriving, doors closing) all sound within earshot of each other, and yet they bear no meaningful harmonic relationship to each other. The mix that results sounds unintentional, technological and out of tune.

The turnstile bank in particular, with its microtonal pitch variations, often sounds like a badly tuned instrument. Since the pitch variation is random and carries no information, we perceive it as mildly grating dissonance. The riders' comments support the notion that while the signals can be informative, they lack aesthetic value; sound in the subway is viewed by riders as entirely utilitarian.

\section{$5 \quad$ Proposal for a New Sound Design}

In this section, we present design alternatives for the system of signals in the New York City subway system. These new designs attempt to improve on the existing design in the following ways:

1. Improved Communication Effectiveness. This is accomplished by using a wider variety of easily distinguished signals with a logical and intuitive mapping to the conditions enumerated in section 2 above.

2. Improved Listenability. This is accomplished through the use of an expanded sound palette and deliberate composition. A variety of timbres, pitches and rhythms will be used to create a soundscape that is harmonic, varied, and textured. The new design employs variations in rhythm, harmony, and counterpoint to create an environment that is more stimulating, more pleasant, and less stressful than the existing design.

The notion of a wind chime, in which distinct tones are struck at unpredictable time intervals, is used to inform the new design. As with a wind chime, the new system contains a group of sounds which sound pleasing individually and in combination with each other.

\subsection{Acoustic Criteria}

Previous studies of auditory signals and warnings [1] provide us with some insights into the design of an effective alert sound. All signals should be constructed of distinctive tonal elements that posses a sharp attack and a rapid decay. The noisy attack and brief tonal body of the sound allow it to be heard distinctly, kept short (reducing overlap and masking), and to be easily localised. Our example uses the sounds of pitched wood bars (marimba) as the basic sonic building block. This sound has a brief, broad-band onset transient that makes it less prone to masking effects than the steady-state tone currently in use [4]. The comparative brevity of the sounds themselves also mitigates masking problems by simply reducing temporal overlap. The proposed turnstile "success" sound, for example, has a half-life of less than $150 \mathrm{~ms}$ and has substantially decayed after $200 \mathrm{~ms}$, compared to a steady state duration of $1000 \mathrm{~ms}$ for the existing "success" signal.

In addition to the selection criteria for individual sound elements, the distribution of the ensemble of tonal, rhythmic and tymbral elements is crucial to the success of the design. Just as an interior designer or architect strives for a unified look when selecting colors and materials, so too must a sound designer select sounds that inform and complement each other and are appropriate for the target space. To this end, different complementary timbres were selected for the train arrival signals (modified flute) and the closing doors signal (bell).

\subsection{Composition}

A new set of mappings of turnstile swipe results to sounds, presented in table 2, yields more effective communication of useful information to riders. The five sounds represent three basic categories, each of which prescribe a different action for the rider. The "modified" success and swipe failure sounds are each variants on their respective themes; in each case, these signals reprise the original signal (to indicate the action to be taken) but then add an element that expresses an additional warning. Additionally, the signals for arriving trains have 
been differentiated as shown in table 3. Example 4: Proposed new signals presents the individual sound elements that make up the proposed new sound design.

\begin{tabular}{|l|l|l|}
\hline Transaction result & Specific user condition & Associated Sound \\
\hline \multirow{4}{*}{ Success } & Token OK & \multirow{2}{*}{ Success } \\
\cline { 2 - 2 } & Metrocard OK & \\
\cline { 2 - 3 } & Metrocard OK - reduced rate & Success (modified) \\
\cline { 2 - 3 } & $\begin{array}{l}\text { Metrocard OK - funds low (one ride or } \\
\text { less remaining on pay-per-use Metrocard) }\end{array}$ & \\
\hline Swipe failure & Swipe again & Swipe failure \\
\cline { 2 - 3 } & Swipe again at this turnstile & Swipe failure (modified) \\
\hline Card failure & Insufficient funds on Metrocard & Card failure \\
\cline { 2 - 3 } & $\begin{array}{l}\text { Monthly, weekly or daily Metrocard } \\
\text { expired }\end{array}$ & \\
\hline
\end{tabular}

Table 2: New mappings of turnstile transactions to audible signals

\begin{tabular}{|l|l|}
\hline Uptown local train arriving & Rising arpeggio, slow \\
\hline Uptown express train arriving & Rising arpeggio, fast \\
\hline Downtown local train arriving & Falling arpeggio, slow \\
\hline Downtown express train arriving & Falling arpeggio, fast \\
\hline
\end{tabular}

\section{Table 3: New mapping of train arrivals to audible signals}

The proposed sound design was developed through an iterative process of composition, simulation and revision. Simulation techniques were crucial to understanding the implications of various design choices. Simulations took into account the frequency distribution of the various signal-causing events in the subway system, including train arrivals and Metrocard swipe outcomes. Figure 2 shows the distribution of 420 Metrocard swipe outcomes as measured at a Manhattan subway station. The amount of time it takes for this many swipes to occur, as well as the number of trains that will arrive and depart in this period, varies widely from station to station and according to the time of day, but the distribution of swipe outcomes is more or less consistent.

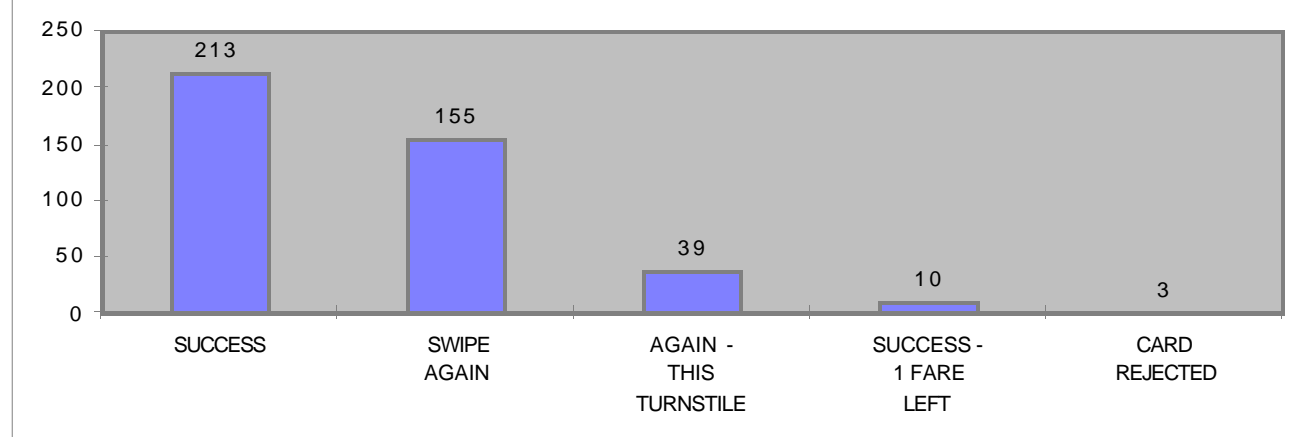

Figure 2: The results of 420 Metrocard swipes

We used Max software to develop a simulation model that takes these distributions into account and allows various parameters to be changed to simulate stations of different sizes and different times of day. Our simulation uses background recordings from actual stations (with turnstile sounds removed), as well as spatialization and reverberation processing of the signals to create a realistic audio field that simulates a bank of turnstiles. Spatialized recordings of the plastic Metrocards being physically swiped, along with the mechanical sounds of the turnstiles cocking forward are coordinated and mixed with the proposed signals to complete the simulation. The simulation helped us to identify the turnstile success sound as the keynote sound in the 
environment; as the most frequently heard signal, it is the sound in relation to which all other sounds in the environment will be heard. The composition was developed around the fundamental C5 pitch of this signal and an implicit C Major chord, which is the key of the design. All the other signals consist of pitches or pitch combinations that either resolve to or naturally modulate toward C Major. Example 5: Simulation of the propposed new subway station presents the ensemble of signals as they might sound in an actual subway station.

\section{Conclusion: Design Guidelines for Audible Information Systems}

Sound design for any environment must be systemic. Because each sound is heard in relation to others, we must learn to design informational sounds in contextual groups so that signals will not mask each other and so that they will be agreeably distributed in frequency, timbre, rhythm, etc.. While an individual signal in isolation might communicate its message effectively, systems involving multiple signals must be carefully designed in order to avoid acoustical interference and communication ambiguities. Even information environments as simple as the subway system require a systemic approach to sound design and strategic mapping of information to signals in order to make the best use of sound.

Audible information signals are generally tonal, and tonal sounds interact musically and are inevitably perceived musically. Recognizing this, we should consider all systems of audible signals to be musical systems, and we should bring to bear the and skills and discipline of composers when designing such systems. In sound as in graphic arts, good creative design results not only in a more attractive product, but in a more effective means of communicating the information at hand.

\section{References}

1. Momtahan, K, Hetu, R., and Tansley, B. Audibility and identification of auditory alarms in the operating room and intensive care unit. Ergomonics. 36, 1993, 1159-1176.

2. Ying K Leung, Sean Smith, Simon Parker and Russell Martin: Learning and Retention of Auditory Warnings. In: ICAD ‘97 Conference Proceedings, 1997.

3. James L. Alty and Paul Vickers: The CAITLIN Auralization System: Hierarchical Leitmotif Design as a Clue to Program Comprehension. In: ICAD ‘97 Conference Proceedings, 1997.

4. Truax, B. Acoustic Communication. Ablex Publishing Corporation, Norwood, NJ, 1984.

5. Schafer,R. M. The Soundscape: Our Sonic Environment and the Tuning of the World, Destiny Books, Rochester, VT, 1977

6. Tufte, E. Visual Explanations. Graphics press, Cheshire, Connecticut, 1997. 